\section{PSICOLOGÍA IBEROAMERICANA}

\section{Psicología Iberoamericana}

ISSN: 1405-0943

psicología.iberoamericana@uia.mx

Universidad Iberoamericana, Ciudad de México

México

Flores Plata, Lorena Alejandra; Cárdenas López, Georgina; Durán Baca, Ximena; de la Rosa Gómez, Anabel

Psicoterapia vía internet: aplicación de un programa de intervención cognitivo-conductual para pacientes con depresión

Psicología Iberoamericana, vol. 22, núm. 1, enero-junio, 2014, pp. 7-15

Universidad Iberoamericana, Ciudad de México

Distrito Federal, México

Disponible en: http://www.redalyc.org/articulo.oa?id=133944229002

Cómo citar el artículo

- Número completo

- Más información del artículo

- Página de la revista en redalyc.org

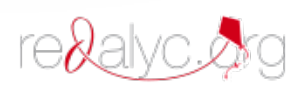

Sistema de Información Científica

Red de Revistas Científicas de América Latina, el Caribe, España y Portugal Proyecto académico sin fines de lucro, desarrollado bajo la iniciativa de acceso abierto 


\title{
Psicoterapia vía internet: aplicación de un programa de intervención cognitivo-conductual para pacientes con depresión
}

\author{
Psychotherapy via internet: Implementing a program of behavioral \\ cognitive intervention for patients suffering depression
}

\author{
Lorena Alejandra Flores Plata ${ }^{1}$ \\ Georgina Cárdenas López \\ Ximena Durán Baca \\ Anabel de la Rosa Gómez \\ Universidad Nacional Autónoma de México
}

\section{RESUMEN}

En México, 28.6\% de la población presentó al menos un trastorno mental alguna vez en su vida y sólo una de cada diez personas que requirieron atención psicológica accedieron a un profesional, lo cual sugiere la incorporación de alternativas que faciliten el acceso a los servicios de salud mental, como psicoterapia vía internet. En este estudio se exponen los hallazgos obtenidos de la aplicación de una intervención cognitivo-conductual vía internet para pacientes con depresión. Se informa de los datos de ocho participantes; seis mujeres y dos hombres, en un rango de edad de 19 a 48 años, con diagnóstico de depresión leve y moderada, evaluada según los criterios del DSM-IV-R. Se evaluó el tamaño del efecto de la intervención y la satisfacción del participante con la modalidad empleada. Los resultados obtenidos muestran la eficacia de la psicoterapia vía internet y su adaptación en un contexto social y cultural como el mexicano.

Palabras clave: tratamiento, depresión, cognitivo-conductual, internet, diseminación.

\section{ABSTRACT}

In Mexico, 28.6\% of the population has suffered a mental disorder once in their lives, and only one in ten people who required psychological care have consulted a professional. This points to the need to implement alternatives for access to mental health services, such as psychotherapy via internet. In this study we present the findings from the application of a behavioral-cognitive intervention via internet for patients with depression. We report on the data of eight participants; six women and two men, between 19 and 48 years old, diagnosed with light and moderate depression, evaluated according to the dsm-iv-r criteria. The size of the intervention's effect was evaluated, as well as the participant's satisfaction with the method used. The results obtained show the effectiveness of psychotherapy via internet and its adaptation in a social and cultural context such as that of Mexico.

Keywords: treatment, depression, behavioral-cognitive, internet, dissemination.

${ }^{1}$ Recibido: 27 de abril de 2013 - Aceptado: 24 de febrero de 2014.

Para correspondencia: Av. Universidad 3004, col. Copilco-Universidad. México, D. F. 04510. Laboratorio de Enseñanza Virtual y Ciberpsicología. Edificio B, Piso 1, Laboratorio 2. E-mail: unam.flores@gmail.com. Tel. (55) 56222292. 


\section{INTRODUCCIÓN}

Según cifras de la Organización Mundial de la Salud (OMs), a través de su Informe sobre la salud en el mundo 2001, cerca de 450 millones de personas han padecido trastornos mentales y de conducta, pronosticando que uno de cada cuatro individuos desarrollará uno o más de éstos en el curso de su vida (oms, 2004).

La oms calculó que el impacto de los trastornos mentales en el mundo representa $13 \%$ de las causas de discapacidad o muerte prematura, y estimó que para el 2020 los trastornos mentales aumentarán hasta contribuir con $15 \%$ de las causas de discapacidad a escala mundial; por ejemplo, la depresión contribuirá con $5.7 \%$ de enfermedades que provocan pérdida de años de vida, ajustados en función de una discapacidad. Estas cifras dan una idea del esfuerzo que se debería llevar a cabo para enfrentar los impactos ocasionados por trastornos mentales (oms, 2004).

En México, se calculó que $28.6 \%$ de la población, sin distinción de sexo, presentó alguna vez en su vida alguno de los 23 trastornos mentales y del comportamiento listados en la Clasificación Internacional de Enfermedades, versión 10 (CIE-10), de los cuales ocupan el lugar de mayor frecuencia (14.3\%) los trastornos de ansiedad, seguidos por trastornos por abuso de sustancias $(9.2 \%)$ y, en tercer lugar, los trastornos afectivos (9.1\%), que incluyen la depresión (Medina-Mora et al., 2003). Específicamente, la prevalencia nacional de depresión en 2004 fue de $4.5 \%$, donde $5.8 \%$ corresponde a las mujeres y $2.5 \%$ a hombres (Belló, PuentesRosas, Medina-Mora \& Lozano, 2005).

Estas cifras cobran relevancia ante el costo social, familiar o personal que implica enfrentar un trastorno mental. Dichos costos no sólo se refieren a los económicos (tanto personales como sociales), sino también a las repercusiones directas e indirectas ocasionadas por la prevalencia de los trastornos mentales, tales como la pérdida de empleo y el impacto en la productividad, a las que se añaden otras consecuencias más difíciles de ponderar, como el efecto negativo del estigma y la discriminación, así como las afectaciones por pérdida de oportunidades de los individuos o familiares (oms, 2004). Por ello, han surgido distintas alternativas para la atención y el acceso a servicios de salud mental, cuyo propósito es aumentar la disponibilidad de los servicios para la población. Entre estas alternativas, se encuentra la implementación de métodos terapéuticos ya existentes a través de herramientas tecnológicas.

La terapia cognitivo-conductual (TCC) ha mostrado ser efectiva y exitosa para el tratamiento de trastornos como la depresión, favoreciendo procedimientos breves centrados en el paciente (Hollon et al., 2005). Sin embargo, el acceso a esta terapia se ha visto obstaculizado por diferentes factores, entre los que destacan la escasez de capital humano capacitado, listas de espera, barreras personales como el estigma, altos costos económicos e inversiones de tiempo importantes (Kohn, Saxena, Levav \& Saraceno, 2004; Wright et al., 2005). Frente a estos factores, se identifica que la incorporación de alternativas terapéuticas basadas en tecnologías, como la psicoterapia vía internet, tiene varias ventajas, como: una amplia oferta de ayuda, acceso remoto, confidencialidad, reducción de la inhibición, atención temprana, experiencia terapéutica, motivación por parte del paciente, entre otras, sin olvidar su eficiencia económica (Clarke et al., 2009; Marks, Cavanagh \& Gega, 2007). Estas características favorecen la disponibilidad en la oferta de servicios y reducen la cantidad de tiempo clínico que hoy en día se invierte en la atención de los trastornos mentales. Hay que señalar que en la aplicación de servicios de salud mental vía internet, todo debe ajustarse a lineamientos éticos que rigen la labor del psicólogo, y específicos para cada área geográfica donde se labora. La psicoterapia vía internet debe ser diseñada y ofrecida por expertos, supervisados por terapeutas clínicos, y realizada sin reducción de las ganancias que la terapia tradicional ofrece (Jarne, 2001; Titov et al., 2010).

Derivados de modalidades terapéuticas con tecnologías como la computadora o el uso de internet, la literatura da cuenta de resultados prometedores, progresivos y de mayor acceso en el tratamiento de: estrés postraumático (Lange et al., 2000); ansiedad y fobias (Botella et al., 2008; Marks, 1999); trastorno obsesivocompulsivo (Greist et al., 1998); depresión no suicida (Osgood-Hynes et al., 1998; Proudfoot et al., 2004; Richards \& Richardson, 2012); tabaquismo y consumo de alcohol (Finfgeld-Connett, 2006; Schneider, Schwartz \& Fast, 1995), entre otros. En general, se sabe que los tratamientos apoyados en tecnologías dan mejores resultados que la ausencia de tratamiento (Andersson \& Cuijpers, 2009) y resultados compara- 
bles con la terapia cognitivo-conductual tradicional (cara a cara), así como en comparación con otros tratamientos alternativos (Marks et al., 2007; Pittaway et al., 2009). La evidencia respalda que la terapia cognitivo-conductual mediada con tecnologías es segura y efectiva (Barak, Hen, Boniel-Nissim \& Shapira, 2008; Graham, Franses, Kenwrit \& Marks, 2000; Jones \& Stokes, 2009; Richards, 2009).

Específicamente, para la depresión existe evidencia de que la psicoterapia vía internet puede ser un método eficaz, tanto en casos leves como severos (Griffiths, Farrer \& Christensen, 2010; Newman, Szkodny, Llera \& Przeworski, 2011; Richards \& Richardson, 2012), y se ha demostrado su eficacia a largo plazo (Ruwaard et al., 2009) y su contribución a eliminar la posibilidad de una recaída después del tratamiento (De Graaf et al., 2009). En algunos casos, la eficacia de esta alternativa se ha hecho presente incluso sin el apoyo o seguimiento directo de un terapeuta clínico (Meyer et al., 2009; Richards \& Timulak, 2012).

En el caso específico de México, no existen antecedentes claros sobre el uso de tecnologías como internet para tratamientos psicológicos. Se han articulado acciones de implementación de tecnología para tratamientos psicológicos en este país motivadas por las experiencias exitosas en el uso de tecnologías en otras naciones. Inicialmente se ha trabajado en estrategias exitosas de entrenamiento en habilidades clínicas para brindar servicios psicoterapéuticos vía internet (Cárdenas, Serrano, Flores \& de la Rosa, 2008), así como en la realización de manuales detallados de intervención para casos de ansiedad y depresión, principalmente (Cárdenas, Flores \& de la Rosa, 2011), que abren líneas de investigación e intervención para propiciar un mayor alcance de servicios en salud mental a través de modalidades tecnológicas.

Los antecedentes internacionales exitosos y los trabajos iniciales de estrategias clínicas con tecnologías en este país, así como la necesidad de alternativas basadas en tecnologías motivan la presente investigación, que tiene como objetivo realizar un estudio exploratorio en población mexicana de un tratamiento cognitivo-conductual ofrecido totalmente vía internet para participantes con sintomatología de depresión de leve a moderada.

\section{MÉTODO}

\section{Participantes}

La muestra se integró por participantes con depresión no severa. La determinación de depresión no severa se realizó con base en los criterios del DSM-IV-R (APA, 2000), complementados con los resultados de los inventarios adaptados de depresión y ansiedad de Beck (Jurado et al., 1998; Robles, Varela, Jurado \& Pérez, 2001). Se aplicaron también los siguientes criterios de exclusión: estar actualmente en tratamiento psicológico o psiquiátrico: abuso de alcohol o drogas, así como comorbilidad con otros trastornos.

La muestra, no probabilística, quedó conformada por seis mujeres (75\%) y dos hombres (25\%); la edad promedio fue de $\mathrm{M}=26.13$ años (D.E. = 9.5) (el participante más joven fue de 19 años y el más grande, de 48 años de edad); siete participantes eran estudiantes de licenciatura y sólo uno estudiaba el bachillerato; seis recibieron atención a través de chat escrito, mientras que uno la recibió por videoconferencia y otro por audio; seis participantes habitaban en el Distrito Federal y dos en el interior de la república, en el estado de Tlaxcala.

\section{Instrumentos}

Para determinar la intensidad de las variables depresión y ansiedad, se empleó el Inventario de Depresión de Beck (BDI) y el Inventario de Ansiedad de Beck (вAI) (para un diagnóstico diferencial, por la comorbilidad que presentan ambos trastornos), en sus versiones adaptadas para México (Jurado et al., 1998; Robles et al., 2001) con un índice de consistencia interna de 0.87 en depresión y de 0.83 en ansiedad. El BDI recorre una escala de 0 a 63, entre mayor es el puntaje mayor es el nivel de depresión. El corte para la muestra fue el intervalo de puntaje de 6 a 30 en la escala de BDI, equivalente a una sintomatología leve o moderada, que implica por lo menos tres síntomas de depresión de intensidad variada.

Se aplicó un cuestionario de satisfacción de usuario respecto del servicio recibido. Este cuestionario se desarrolló para población que recibe tratamiento vía internet y consta de 25 preguntas de respuesta "SÍ" o "NO", separadas por los siguientes apartados: requerimientos, que abarca aspectos de logística sobre los 
requerimientos de software, hardware y las instrucciones iniciales generales de conexión a la terapia; programa de psicoterapia vía internet, que evalúa si el programa de psicoterapia por esta vía se adecuó a las necesidades del participante, si los datos del tratamiento fueron proporcionados y clarificados, así como si el tiempo dedicado al tratamiento se consideró suficiente; medio virtual de la intervención psicológica, que evalúa principalmente la facilidad del usuario por el medio utilizado (internet); y, la comunicación con el terapeuta, que evalúa frecuencia, comunicación, apoyo y claridad.

Todos los participantes firmaron un formato de consentimiento, el cual incluía la información del tratamiento que recibirían, así como los lineamientos respecto a procedimientos administrativos.

\section{Procedimiento}

A partir de la difusión realizada en los servicios de comunicación interna del campus de la Universidad Nacional Autónoma de México, así como en el Centro de Servicios Psicológicos (CSP), de la Facultad de Psicología, se ofreció el servicio de psicoterapia vía internet. En la fase inicial de contacto, los participantes solicitaban servicio de psicología a través del CSP y este Centro los canalizaba al programa de tratamiento vía internet; en ese primer contacto se identificaba si los candidatos reportaban síntomas de depresión y si contaban con los elementos tecnológicos que posibilitaran su conexión a las sesiones posteriores. Aquéllos con estas características eran invitados a recibir un tratamiento vía internet, en sesiones semanales de una hora, durante 16 semanas. Los participantes seleccionados recibieron por correo electrónico el BDI y el BAI. Por la misma vía se establecieron el día y la hora de la primera sesión de evaluación, la cual se efectuó necesariamente por videoconferencia. El tratamiento estuvo basado en el programa de intervención de Cárdenas et al. (2011) para tratamientos vía internet en depresión. El procedimiento de la aplicación se organizó en cinco etapas: fase inicial de contacto, evaluación inicial, tratamiento, evaluación final y seguimiento.

En la evaluación inicial, por videoconferencia, se explicaron las características del tratamiento, se resolvieron dudas y se aplicó una entrevista de screening, cuyo objetivo fue descartar la existencia de cualquier otro trastorno mental antes de confirmar su participación. Con los participantes de la muestra se acordaban el día y la hora, así como la vía de comunicación (chat, audio o videoconferencia) de las sesiones. Para las sesiones de evaluación y de tratamiento, fue importante solicitarles un espacio privado libre de ruidos, donde pudieran estar por lo menos una hora una vez a la semana, durante el lapso del tratamiento. La intervención se orientó principalmente a lograr una mejoría en el participante, respecto a la sintomatología inicial y motivo de consulta, a través de un protocolo de TCC para depresión (Cárdenas et al., 2011).

El programa de intervención es de corta duración, de participación activa, focalizado y directivo, en 16 sesiones semanales de una hora. Los principales componentes utilizados y el orden de uso fueron: 1) reestructuración cognitiva, 2) identificación y expresión de emociones, 3) trabajo con autoestima y asertividad, 4) solución de problemas, y 5) cierre; contemplando evaluaciones pre y post. Como apoyo a la sesión, el paciente realiza lecturas, registros de pensamientos y emociones, y tiene un seguimiento semanal de mejoría.

Al final del tratamiento se valoró la sintomatología y satisfacción del participante, respecto a la evaluación inicial y con un seguimiento a seis meses. Los resultados de las evaluaciones se exponen en el siguiente apartado, considerando para los resultados clínicos, la comparación entre medidas pre, post y seguimiento, así como el tamaño del efecto individual de tratamiento otorgado.

\section{RESULTADOS}

Con la finalidad de establecer comparaciones entre las evaluaciones pre, post y seguimiento, y tomando en cuenta el tamaño de la muestra, se aplicó la prueba no paramétrica Wilcoxon para muestras relacionadas, a fin de conocer si hubo cambios en los participantes atendidos. A partir de este cálculo, puede afirmarse que existen diferencias significativas para las medidas. La medida de depresión pasa de una media 19.87 a 4.5, lo que muestra una mejoría significativa $(\mathrm{Z}=-2.524$, $\mathrm{p}=0.012)$, mientras que la medida de ansiedad pasa de una media de 13.75 a 5.13, mostrando una mejoría significativa $(Z=-2.176, p=0.03)$. Respecto al seguimiento a seis meses, cuyas medias fueron de 5.6 para depresión $(\mathrm{Z}=-1.192, \mathrm{p}=0.233)$, y 5.38 para ansiedad 
$(\mathrm{Z}=-.141, \mathrm{p}=0.888)$, tal como se observa en la tabla 1 , se mantuvieron las ganancias clínicas del tratamiento, ya que no existen diferencias significativas respecto a las medidas postratamiento. Este mantenimiento de ganancias es positivo, en tanto que implica que los participantes adquirieron herramientas durante la intervención que pueden seguir replicando para nuevas situaciones y que les permiten continuar con los beneficios obtenidos en el tratamiento.

Con la intención de conocer el efecto de la intervención por participante, así como los resultados obtenidos de la prueba de Wilcoxon, se aplicó una prueba interna del Tamaño del Efecto Individual (TEI), con la cual se establece si existen cambios clínicos significativos individuales, valorados a partir de la división de cada diferencia pre-post entre la desviación típica del pretratamiento. Tal como señalan Pardo y Ferrer (2013), este cociente debe ser alrededor de 0.80 o mayor para representar un cambio clínicamente significativo, tal como se aplicó en este trabajo.

Para la evaluación de depresión los cambios clínicos fueron significativos grandes en siete participan- tes y en uno más fueron significativos moderados; el paciente con cambios moderados es el que presentó inicialmente las medidas más bajas y cuyas posibles mejorías eran muy acotadas. Para el caso del seguimiento, respecto a la evaluación posterior, al menos tres participantes continuaron disminuyendo sus niveles, uno más se mantuvo y cuatro más elevaron su sintomatología sin volver a la escala inicial de su evaluación pretratamiento. En el caso de la evaluación de ansiedad, a excepción de un participante, los otros siete obtuvieron ganancias clínicas significativas, respecto a sus evaluaciones pre-post, cinco con un cambio significativo grande y tres más con un cambio moderado; en el caso del seguimiento, cinco participantes continúan disminuyendo su sintomatología, mientras tres más elevan sus índices a los seis meses, tan sólo uno eleva su condición superando la evaluación previa, pero manteniéndose en una escala mínima; los otros dos elevan su evaluación sin llegar o superar la inicial (ver tabla 2).

Tabla 1. Grado de cambio pretest-postest observado después de la intervención vía internet (Prueba Wilcoxon) $(\mathrm{N}=8$ )

\begin{tabular}{c|cc|cc|c|c}
\hline \multirow{2}{*}{ Variable } & \multicolumn{3}{|c|}{ Pre } & \multicolumn{2}{c|}{ Post } & \multicolumn{2}{c}{ Z } & Significancia \\
& \multicolumn{2}{|c|}{ Media } & DT & Media & DT & \\
\hline Ansiedad & 13.75 & 8.102 & 5.14 & 3.182 & -2.176 & 0.03 \\
\hline Depresión & 19.88 & 8.560 & 4.50 & 2.204 & -2.524 & 0.012 \\
\hline
\end{tabular}

Tabla 2. Tamaño del Efecto Individual (TEI) en evaluaciones pre-post y un seguimiento a seis meses, en BDI y BAI ( $N=8$ )

\begin{tabular}{|c|c|c|c|c|c|c|c|c|c|c|}
\hline \multirow{2}{*}{$P$} & \multicolumn{5}{|c|}{ Evaluación depresión (BDI) } & \multicolumn{5}{|c|}{ Evaluación ansiedad (BAI) } \\
\hline & Pre & Post & $\begin{array}{c}\text { TEI } \\
\text { Pre-Post }\end{array}$ & Seg. & $\begin{array}{c}\text { TEI } \\
\text { Post Seg. }\end{array}$ & Pre & Post & $\begin{array}{c}\text { TEI } \\
\text { Pre-Post }\end{array}$ & Seg. & $\begin{array}{c}\text { TEI } \\
\text { Post Seg. }\end{array}$ \\
\hline 1 & 24 & 5 & $2.22 *$ & 3 & $0.91^{*}$ & 22 & 7 & $1.75^{*}$ & 10 & -0.94 \\
\hline 2 & 28 & 4 & $2.80^{*}$ & 3 & 0.45 & 24 & 5 & $2.22 *$ & 2 & $0.94^{*}$ \\
\hline 3 & 8 & 2 & 0.70 & 0 & $0.91 *$ & 11 & 3 & $0.93 *$ & 2 & 0.31 \\
\hline 4 & 30 & 5 & $2.92 *$ & 8 & -1.36 & 7 & 3 & 0.47 & 2 & 0.31 \\
\hline 5 & 10 & 4 & 0.70 & 9 & -2.27 & 16 & 9 & $0.82^{*}$ & 14 & -1.57 \\
\hline 6 & 12 & 5 & $0.82^{*}$ & 7 & -0.91 & 21 & 2 & $2.22^{*}$ & 1 & 0.31 \\
\hline 7 & 24 & 2 & $2.57^{*}$ & 2 & 0 & 3 & 2 & 0.12 & 4 & -0.63 \\
\hline 8 & 23 & 9 & $1.64^{*}$ & 13 & -1.81 & 6 & 10 & -0.47 & 8 & 0.63 \\
\hline
\end{tabular}

Nota: $\mathrm{P}=$ Participantes; $\mathrm{BDI}=$ Inventario de Depresión de Beck; $\mathrm{BAI}=$ Inventario de Depresión de Ansiedad; TEl = Tamaño del Efecto Individual ${ }^{*} \geq 0.80$. 
En síntesis, se observan cambios positivos a partir de la intervención realizada, que revelan una influencia significativa del tratamiento brindado sobre los participantes tratados, así como de las técnicas que se incorporaron en el tratamiento, las cuales se conservan a lo largo del tiempo como un aprendizaje que favorece el mantenimiento de la mejoría.

Los porcentajes de satisfacción expresados por los participantes fueron altos $(M=94.50$, D.E. $=9.78)$. En general, los participantes evaluaron de manera favorable los cuatro aspectos de esta área: requerimientos, programa de psicoterapia vía internet, medio virtual de la intervención psicológica y la comunicación con el terapeuta. Finalmente, los participantes en su totalidad expresaron que recomendarían el servicio de psicoterapia vía internet a algún familiar o amigo.

\section{DISCUSIÓN}

El presente estudio cuantitativo tuvo como propósito poner a prueba la efectividad de un tratamiento cognitivo-conductual para brindar sevicios de salud mental, en la modalidad vía internet a un grupo de participantes con sintomatología de depresión leve y moderada.

Los resultados obtenidos afirman que existen diferencias significativas en los ocho participantes en las medidas de depresión y ansiedad pre, post, así como mantenimiento de las ganancias en su seguimiento; en la evaluación de depresión, se alcanzó un tamaño del efecto individual significativo para siete de los ocho participantes y en uno más, el que presentó menor medida de depresión, se reconoce un efecto significativo moderado. Es decir, los pacientes atendidos con esta modalidad recibieron un tratamiento eficaz para el diagnóstico de depresión. La modalidad de tratamiento vía internet no representó ningún impedimento y facilitó el acercamiento de un servicio clínico de calidad, lo que apoya los resultados obtenidos en otras investigaciones (Barak et al., 2008; Graham et al., 2000; Jones \& Stokes, 2009; Richards, 2009). Los logros obtenidos en esta investigación muestran la efectividad de la psicoterapia vía internet y la viabilidad de su incorporación en un contexto social y cultural como el mexicano. Con base en la satisfacción percibida de los participantes, así como en futuras investigaciones, será posible hacer los ajustes pertinentes para ofrecer servicios de salud mental cada vez más eficientes.
Para los participantes de este estudio, con diagnóstico de depresión leve y moderada, resultó positiva la idea central de que la incorporación de alternativas terapéuticas basadas en tecnologías posee varias ventajas, como las señaladas por otros investigadores (Clarke et al., 2009; Marks et al., 2007), por ejemplo: una amplia oferta de ayuda, acceso remoto, confidencialidad, reducción de la inhibición, atención temprana, experiencia terapéutica, motivación por parte del paciente, entre otros, sin olvidar su eficiencia económica, lo que confirma los hallazgos encontrados para este tipo de modalidades (Griffiths et al., 2010; Richards \& Richardson, 2012; Newman et al., 2011). Para el caso de la implementación de la "telesalud", numerosos proyectos han demostrado que las intervenciones preventivas y terapéuticas por modalidades como la videoconferencia o el chat, pueden ser tan efectivas como las intervenciones presenciales cara a cara, por ejemplo para el tratamiento de niños con depresión (Nelson, Barnard \& Cain, 2006); para agorafobia con trastorno de pánico (Allard et al., 2007; Bouchard et al., 2000); para depresión (Meyer et al., 2009; Osgood-Hynes et al., 1998; Perini, Titov \& Andrews, 2009; Richards, 2009; Ruwaard et al., 2009); depresión y ansiedad (Griffiths et al., 2010), o bien, en casos de tabaquismo y consumo de alcohol (FinfgeldConnett, 2006; Schneider et al., 1995).

Resulta sorprendente que a pesar de la gran necesidad de contar con una mayor cobertura de servicios de salud mental en nuestro país (Medina-Mora et al., 2003), esta herramienta no ha sido identificada claramente por las instituciones gubernamentales encargadas de brindar apoyo y tratamiento en este sector y que, a pesar de las numerosas investigaciones con hallazgos positivos y la utilidad reportada, esta modalidad no haya sido adoptada ampliamente en nuestro país, pese a las ventajas implicitas, en particular, en las localidades rurales e indigenas distantes, donde estos servicios son incipientes.

Destacan varios beneficios en la incorporación de servicios de psicología apoyados con tecnología, como la reducción de tiempos y costos, tanto para los terapeutas como para los pacientes; contar con materiales de psico-educación y ejercicios para los participantes para su acceso desde un dispositivo móvil, en cualquier lugar y sin necesidad de movilidad física (Clarke et al., 
2009; Marks et al., 2007). Otro objetivo importante de estos servicios de salud mental, de acuerdo con Vallejo y Jordán (2007), es que la reducción del tiempo dedicado a un tratamiento, por lo general favorecerá su cumplimiento para obtener resultados favorables, lo que incrementa la adherencia terapéutica.

Los servicios terapéuticos vía internet son una alternativa de tratamiento para personas con deseos de tomar o servirse de una psicoterapia, y que por cualquier motivo no han podido lograrlo, incluso obstaculizados por la misma sintomatología de su trastorno - como la depresión-, o bien, un servicio para personas familiarizadas y motivadas por el uso de tecnologías, lo que debilita el obstáculo de la escasez de capital humano capacitado y los altos costos económicos (Wright et al., 2005).

Cabe destacar algunas limitaciones inherentes al estudio: primero, el tamaño de la muestra, que se debió en gran parte a que diversos participantes no acepta-

\section{REFERENCIAS}

Allard, M., Bouchard, S., Marchand, A., Cournoyer, L., Green-Demers, I. \& Renaud, P. (2007). L’efficacité de la psychothérapie pour le trouble panique en videoconference: réplication et alliance thérapeutique. Revue Québécoise de Psychologie, 28(2), 43-64.

American Psychiatric Association (2002). Manual diagnóstico y estadístico de los trastornos mentales-IV-TR. Barcelona: Masson.

Andersson, G. \& Cuijpers, P. (2009). Internet-based and other computerized psychological treatments for adult depression: a meta-analysis. Cognitive Behaviour Therapy, 38(4), 196-205.

Barak, A., Hen, L., Boniel-Nissim, M. \& Shapira, N. (2008). A comprehensive review and a meta-analysis of the effectiveness of internet-based psychotherapeutic interventions. Journal of Technology in Human Services, 26(2), 109-160.

Belló, M., Puentes-Rosas, E., Medina-Mora, M. \& Lozano, R. (2005). Prevalencia y diagnóstico de depresión en población adulta en México. Salud Pública de México, 47(1), 1-11.

Botella, C., Quero, S., Baños, R. M., García-Palacios, A., Bretón-López, J., Alcaniz, M. \& Fabregat, S. ron el tratamiento, ya que eran enviados para acortar la lista de espera del Centro de Servicios Psicológicos de la Facultad de Psicología, y no habían solicitado esta modalidad de tratamiento como primera opción; segundo, no se ofreció a los participantes la opción de elegir vía de comunicación para recibir su tratamiento, pudiendo ser chat, audio o videoconferencia.

En resumen, se puede concluir que la psicoterapia vía internet está en constante cambio y crecimiento, en la medida en que la tecnología sigue avanzando. La tarea pendiente es dar cuenta de los rendimientos, fallas y aciertos en la aplicación de estas nuevas tecnologías en el tratamiento de trastornos psicológicos, así como centrar estudios en las características de la comunicación no verbal que se da en un proceso terapéutico vía internet, para incluir en él las modificaciones necesarias y potenciar lo que, hasta el momento, haya tenido rendimientos positivos en la disciplina.

(2008). Telepsychology and self-help: the treatment of phobias using the internet. CyberPsychology and Behavior, 11(6), 659-664.

Bouchard, S., Payeur, R., Rivard, V., Allard, M., Paquin, B., Renaud, P. \& Goyer, L. (2000). Cognitive behavior therapy for panic disorder with agoraphobia in videoconference: preliminary results. CyberPsychology \& Behavior, 3(6), 999-1007.

Cárdenas, G., Flores, L. \& De la Rosa, A. (2011). Una alternativa a distancia: psicoterapia vía internet. México: UNAM.

Cárdenas, G., Serrano, B., Flores, L. \& De la Rosa, A. (2008). Etherapy: a training program for development of clinical skills in distance psychotherapy. Journal of Technology in Human Services, 26(1), 470-483.

Clarke, G., Kelleher, C., Hornbrook, M., Debar, L., Dickerson, J. \& Gullion, C. (2009). Randomized effectiveness trial of an Internet, pure self-help, cognitive behavioral intervention for depressive symptoms in young adults. Cognitive Behavior Therapy, 38(4) 222-234.

De Graaf, L. E., Gerhards, S. A., Arntz, A., Riper, H., Metsemakers, J. F., Evers, S. M. \& Huibers, M. J. 
(2009). Clinical effectiveness of online computerized cognitive-behavioural therapy without support for depression in primary care: randomised trial. British Journal Psychiatry, 195(1) 73-80.

Finfgeld-Connett, D. (2006). Web-based treatment for problem drinking. Journal of Psychosocial Noursing, 44(9), 20-27.

Graham, C., Franses, A., Kenwrit, M. \& Marks, I. (2000). Psychotherapy by computer: a postal survey responders to teletext article. Psychiatric Bulletin 2000, $25,12-14$.

Greist, J. H., Marks, I. M., Baer, L., Parkin, J. R., Manzo, P. A., Mantle, J. M. \& Forman, L. (1998). Self-treatment for obsessive-compulsive disorder using a manual and a computerized telephone interview: a US-UK study. MD Computing, 15(3), 149-57.

Griffiths, K. M., Farrer, L., \& Christensen, H. (2010). The efficacy of internet interventions for depression and anxiety disorders: A review of randomized controlled trials. Medical Journal Australia, 7, 4-11.

Hollon, S. D., DeRubeis, R. J., Shelton, R. C., Amsterdam, J. D., Salomon, R. M., O’Reardon, J. P. \& Gallop, R. (2005). Prevention of relapse following cognitive therapy vs. medications in moderate to severe depression. Archives of General Psychiatry, 62(4), 417-422.

Jarne, A. (2001). Hacia un código deontológico de la intervención psicológica a través de internet. Anuario de Psicología, 32(2), 117-126.

Jones, G. \& Stokes, A. (2009). Online counselling: a handbook for practitioners. Londres: Palgrave Macmillan.

Jurado, S., Villegas, M., Méndez, L., Rodríguez, F., Loperena, V. \& Varela, R. (1998). La estandarización del Inventario de depresión de Beck para los residentes de la Ciudad de México. Salud Mental, 21(3), 26-31.

Kohn, R., Saxena, S., Levav, I. \& Saraceno, B. (2004). The treatment gap in mental health care. Bulletin of the World Health Organization, 82, 858-866.

Lange, A., Schrieken, B., Van de Ven, J., Bredeweg, B., Emmelkamp, P., Van der Kolk, J. \& Reuvers, A. (2000). Interapy: the effects of a short protocolled treatment of post-traumatic stress and pathological grief through the internet. Behavioral and Cognitive Psychotherapy, 28(2), 175-192.
Marks, I. (1999). Computer aids to mental health care. Canadian Journal of Psychiatry, 44(6), 548-555.

Marks, I., Cavanagh, K. \& Gega, L. (2007). Hands-on help computer-aided psychotherapy. New York: Psychology Press.

Medina-Mora, M., Borges, G., Muñoz, C., Benjet, C., Jaimes, J., Bautista, C. \& Aguilar-Gaxiola, S. (2003). Prevalencia de trastornos mentales y uso de servicios: resultados de la Encuesta Nacional de Epidemiología Psiquiátrica en México. Salud Mental, 26(4), 1-4.

Meyer, B., Berger, T., Caspar, F., Beevers, C., Andersson, G. \& Weiss, M. (2009). Effectiveness of a novel integrative online treatment for depression (deprexis): randomized controlled trial. Journal of Medical Internet Research, 11(2). Recuperado de www.jmir. org/2009/2/e15/ doi:10.2196/jmir.1151.

Nelson, E., Barnard, M. \& Cain, S. (2006). Feasibility of teletherapy for childhood depression. Counseling and Psychotherapy Research, Special Technology Edition, 6(3), 191-195.

Newman, M. G., Szkodny, L. E., Llera, S. J. \& Przeworski, A. (2011). A review of technology-assisted self-help and minimal contact therapies for anxiety and depression: is human contact necessary for therapeutic efficacy? Clinical Psychology Review, 31(1), 89-103.

Organización Mundial de la Salud (2004). Promoción de los trastornos mentales: intervenciones efectivas $y$ opciones de políticas: Informe compendiado. Ginebra, Suiza: oms.

Osgood-Hynes, D., Greist, J., Marks, I., Baer, L., Heneman, S., Wenzel, K. \& Vitse, H. (1998). Self-administered psychotherapy for depression using a telephoneaccessed computer system plus booklets: an open US-UK study. Journal of Clinical Psychiatry, 59(7), 358-365.

Pardo, A. \& Ferrer, R. (2013). Significancia clínica: falsos positivos en la estimación del cambio individual. Anales de Psicología, 29(2), 301-310.

Perini, S., Titov, N. \& Andrews, G. (2009). Clinicanassisted internet-based treatment is effective for depression: randomized controlled trial. Australian and New Zeeland Journal of Psychiatry, 43(6), 571-578.

Pittaway, S., Cupitt, C., Palmer, D., Arowobusoye, N., Milne, R., Holttum, S., Pezet, R. \& Patrick, H., (2009). Comparative, clinical feasibility study of three tools 
for delivery of cognitive behavioural therapy for mild to moderate depression and anxiety provided on a self-help basis. Mental Health in Family Medicine, 6(3), 145-154.

Proudfoot, J., Ryden, C., Everitt, B., Shapiro, D., Goldberg, D., Mann, A. \& Gray, J. (2004). Clinical efficacy of computerized cognitive-behavioural therapy for anxiety and depression in primary care: randomized controlled trial. The British Journal of Psychiatry, 185, 46-54.

Richards, D. \& Richardson, T. (2012). Computer-based psychological treatments for depression: a systematic review and meta-analysis. Clinical Psychology Review, 332(4), 329-342.

Richards, D. \& Timulak, T. (2012). Satisfaction with therapist-delivered vs. self-administered online cognitive-behavioural treatments for depression in college students. British Journal of Guidance and Counselling, 41(2), 193-207.

Richards, D. (2009). Features and benefits of online counselling: Trinity College online mental health community. British Journal of Guidance \& Counselling, 37(3), 231-242.

Robles, R., Varela, R., Jurado, S. \& Pérez, F. (2001). Versión mexicana del Inventario de ansiedad de Beck: propiedades psicométricas. Revista Mexicana de Psicología, 8, 211-217.
Ruwaard, J., Schrieken, B., Schrijver, M., Broeksteeg, J., Dekker, J., Vermeulen, H. \& Lange, A. (2009). Standardized web-based cognitive behavioral therapy of mild to moderate depression: a randomized controlled trial with a long-term follow-up. Cognitive Behaviour Therapy, 4(38), 206-21.

Schneider, S. J., Schwartz, M. D. \& Fast, J. (1995). Computerized, telephone-based health promotion: smoking cessation program. Computers in Human Behavior, 1(11), 135-148.

Titov, N., Andrews, G., Davies, M., McIntyre, K., Robinson, E. \& Solley, K. (2010). Internet treatment for depression: a randomized controlled trial comparing clinician vs. technician assistance. PLOS One, 5(6). Recuperado de www.plosone.org/article/ info\%3Adoi\%2F10.1371\%2Fjournal.pone.0010939 \#pone-0010939-g001. DOI: 10.1371/journal.pone. 0010939.

Vallejo, M. \& Jordán, C. (2007). Psicoterapia a través de internet. Recursos tecnológicos en la práctica de la psicoterapia. Boletín de Psicología, 91, 27-42.

Wright, J. H., Wright, A. S., Albano, A., Basco, M. R., Goldsmith, L. J., Raffield, T. \& Otto, M. V. (2005). Computer-assisted cognitive therapy for depression: maintaining efficacy while reducing therapist time. The American Journal of Psychiatry, 162(6), 11581164. 